\title{
Determination of Fragmentation Schemes and Metabolites of Fluorinated Histone Deacetylase Inhibitors for Use as Positron Emission Tomography Imaging Agents Using HPLC-MS/MS
}

\author{
Wei-Hsi Chen ${ }^{*}$, Yu-Chieh Hsiao', Ming-Hsin Li², Mu-Chen Tsai1, Chun-Fang Feng2, \\ Han-Chih Chang2, Hung-Wen Yư ${ }^{2}$, Chyng-Yann Shiue ${ }^{3,4}$ \\ ${ }^{1}$ Chemistry Division, Institute of Nuclear Energy Research, Taiwan \\ ${ }^{2}$ Isotope Application Division, Institute of Nuclear Energy Research, Taiwan \\ ${ }^{3}$ PET Center, Department of Nuclear Medicine, National Taiwan University Hospital, Taiwan \\ ${ }^{4}$ PET Center, Department of Nuclear Medicine, Tri-Service General Hospital, Taiwan \\ Email: *whchen@iner.gov.tw
}

How to cite this paper: Chen, W.-H., Hsiao, Y.-C., Li, M.-H., Tsai, M.-C., Feng, C.-F., Chang, H.-C., Yu, H.-W. and Shiue, C.-Y. (2018) Determination of Fragmentation Schemes and Metabolites of Fluorinated Histone Deacetylase Inhibitors for Use as Positron Emission Tomography Imaging Agents Using HPLC-MS/MS. International Journal of Analytical Mass Spectrometry and Chromatography, 6, 1-19.

https://doi.org/10.4236/ijamsc.2018.61001

Received: January 26, 2018

Accepted: March 27, 2018

Published: March 30, 2018

Copyright $\odot 2018$ by authors and Scientific Research Publishing Inc. This work is licensed under the Creative Commons Attribution-NonCommercial International License (CC BY-NC 4.0). http://creativecommons.org/licenses/by-nc/4.0/ (c) (i) \& Open Access

\begin{abstract}
High performance liquid chromatography coupled with tandem mass spectrometry was developed and validated as a method for the analysis of fluorinated histone deacetylase inhibitors (F-HDACi), and then employed to study their metabolism in biosystems. Four HDACi analogs labeled with the positron emission nuclide ${ }^{18} \mathrm{~F}$ constitute a group of potential positron emission tomography imaging agents, which were developed by the Institute of Nuclear Energy Research (INER) and coded as INER-1577 \#1, \#2, \#3, and \#4 during animal studies for the diagnosis of dementia. The performance of the method was found to be suitable for the determination of analog \#3, and it was employed to determine the structures and fragmentation mechanisms of all four analogs and to study the biotransformations of analogs \#3 and \#4. The results indicated that the method used for the determination of analog \#3 was suitable for determining the abundance of the analogs in chemical and biochemical tests with high precision, accuracy, reproducibility, and recovery. Weaknesses in the chemical bonding of the analogs were found to involve the fluoro, dimethylamino, and benzamide groups in a fragmentation mechanism deduced via tandem mass spectrometry. The metabolites of analogs \#3 and \#4 in rat liver microsomes and rat plasma were also identified to clarify their characteristic behaviors in biosystems. The major product of analogs \#3 in liver mi-
\end{abstract}


crosomes was produced by hydroxylation of the benzylic carbon atom, but in rat plasma the metabolites of analog \#3 were produced by hydrolysis of the benzamide group to give a diaminobiphenyl compound with the simultaneous replacement of a fluorine atom by a hydroxyl group. The metabolites of ana$\log \# 4$ in liver microsomes were produced by hydroxylation of the benzylic carbon atom and hydrolysis of the benzamide bond. The results of the studies characterized the chemical and biochemical behaviors of the series F-HADCi analogs.

\section{Keywords}

Histone Deacetylase Inhibitors, Positron Emission Tomography Imaging Agent, Dementia, LC-Tandem MS, Fragmentation Mechanism, Metabolism Pathways, Epigenetics

\section{Introduction}

Progressive central neurodegenerative diseases and symptoms of cognitive loss affect more than $20 \%$ of people with ages of greater than 65 years worldwide, which lead to partial cognitive impairment or various levels of dementia [1]. Because of the high prevalence of these conditions and the resulting heavy burden on patients' families and serious social problems, the development of an early stage diagnostic method is critical for mitigating patients' conditions, in preference to characterization at a late stage. Molecular imaging techniques, including the application of radiotracers in positron emission tomography (PET) and single-photon emission computed tomography [2] [3] [4], which provide clear, unambiguous, and detailed diagnostic images of the central nervous system (CNS), are the most powerful tools for assessing the phases of CNS diseases. The development of highly specific and sensitive imaging markers for various CNS diseases is an essential component of imaging diagnosis [4].

Histone deacetylase (HDAC) and histone acetyl transferase (HAT), which are key enzymes that govern the binding of acetyl groups to histone and adjust gene expression, have been shown to be involved in numerous types of cancer and neurodegenerative disorder [5]. A few studies indicated that HDAC inhibitors could treat cancer [6] [7] [8] and also improve memory [8], which was explained in terms of epigenetics theories as follows [9] [10]. Gene expression and cell proliferation are regulated by the remodeling of chromatin, whereby the DNA complex is wrapped around histone or unwrapped, while histone is acetylated by HATs or acetyl groups are removed by HDACs. This indicated that memory ability is positively related to the degree of acetylation of lysine, which is the terminal amino acid of histone, to create acetyl-lysine. This was concluded from the inhibition of the activity of HDACs with HDAC inhibitors (HDACis), which led to an increase in acetylation. HDACis were suggested to act as neuroprotectors by enhancing synaptic plasticity and cognition ability in cases of neurode- 
generation and cognitive impairment, such as Alzheimer's disease (AD) [11]. The inhibition of the activity of HDACs is more efficient than the enhancement of the activity of HATs in regulating the status of chromatin and improving learning ability [11] [12]. A few drug candidates in animal models of AD were found to reverse learning and memory deficits, such as suberoylanilide hydroxamic acid (SAHA) [13], tubacin [14], and tubastatin [15]. Owing to the specific and critical role of HDACis in $\mathrm{AD}$ patients, the development of PET imaging agents that utilize an HDACi mechanism to visualize pathogenesis in the brains of patients with mild cognitive impairment or AD is particularly interesting [16]. For example, 6-([ $\left.{ }^{18} \mathrm{~F}\right]$ fluoroacetamido)-1-hexanoicanilide, also known as ${ }^{18}$ F-FAHA, which is a radio-fluoro labeled SAHA analog, has been used as an imaging agent [17]. On the other hand, containing benzamide group compounds as HDAC inhibitors, such as mocetinostat, MGCD0103 and entinostat, MS-275 showed the potentials to therapy for cancer were under clinical trials [18].

A series of four target analog HDAC inhibitors containing benzamide groups and fluoro substituents were designed, and their synthesis processes were developed. The analogs were patented by the Institute of Nuclear Energy Research (INER) and coded as INER-1577 \#1 to INER-1577 \#4 during animal studies of $\mathrm{AD}$ [19] [20]. The structures and molecular data of the four INER-1577s are shown in Figure 1, which illustrates their common features, namely, amino-biphenyl or aminopyridyl-phenyl and benzamido groups. Information on the metabolic pathways of xenobiotics throughout the entire biological system is
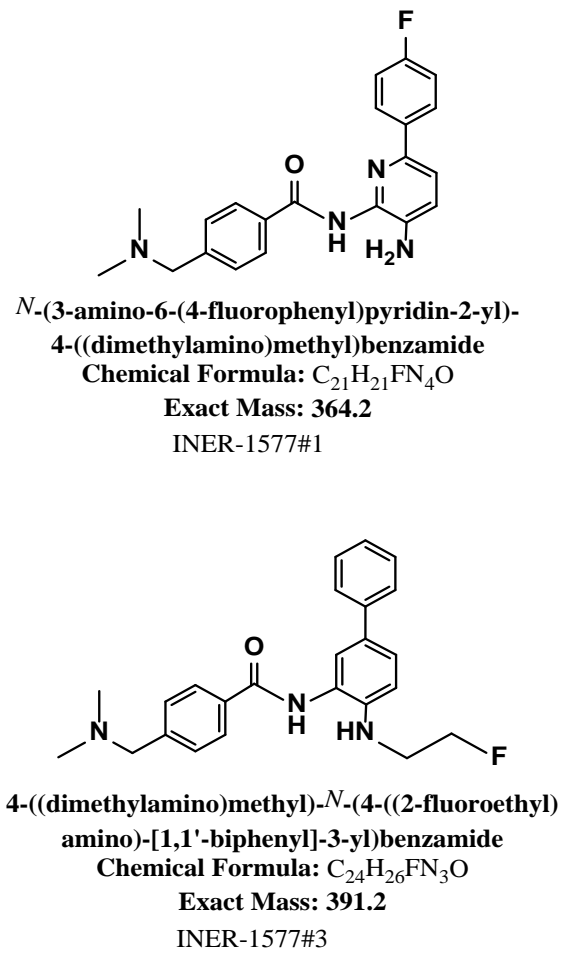

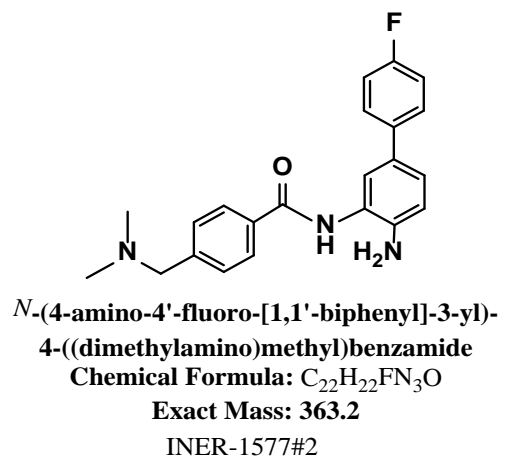

INER-1577\#2

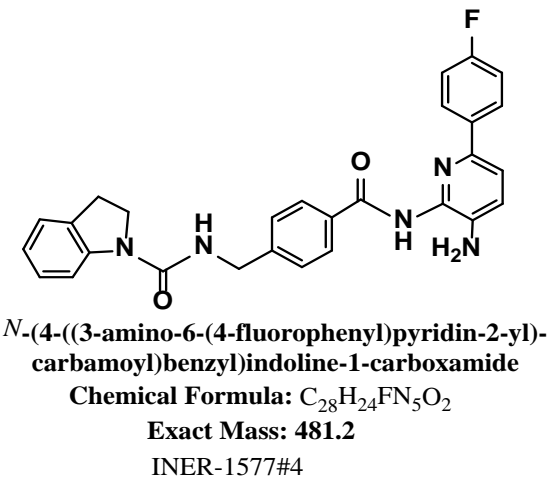

Figure 1. Structures and molecular data of the four INER-1577s. 
crucial for the elucidation of the degradation routes of potentially toxic substances and drug safety [21]. Hence, the chemical and biochemical characteristics of the INER-1577s, including the purities of the prepared materials, fragmentation mechanisms, and biotransformation routes, are vital criteria when deciding how to use the compounds appropriately or modify their structure to enhance their performance.

In this study, a C18 column using gradient elution with aqueous ammonium acetate/methanolic phase that was coupled with a tandem mass spectrometer was set up and validated for the determination of INER-1577 \#3. This high performance liquid chromatography-tandem mass spectrometry (HPLC-MS/MS) method was employed to determine the synthesized purities of INER-1577 \#1, $\# 2$, \#3 and \#4, study the fragmentation schemes of the four analogs, and investigate the biotransformation mechanisms of INER-1577 \#3 and \#4 after incubation with biosystems.

\section{Materials and Methods}

\subsection{Materials and Reagents}

Analytical-grade chemicals for LC-MS were used as received without further purification. Methanol (HPLC and MS grade), ammonium acetate, and dimethyl sulfoxide (DMSO) were all purchased from Merck (Darmstadt, Germany). Ultrapure water (total organic carbon $<5 \mathrm{ppb}$, resistivity $\geq 18.2 \mathrm{M} \Omega-\mathrm{cm}$ at $25^{\circ} \mathrm{C}$ ) was obtained using a Smart DQ3 reverse osmosis reagent water system (Merck Millipore, Billerica, MA, USA) fitted with a $0.22 \mu \mathrm{m}$ polyvinylidene fluoride (PVDF) filter and an ultraviolet (UV) light source. The four patented HDAC inhibitor analogs (INER-1577 \#1 to \#4) were custom prepared by WuXi-AppTec (Shanghai, China), and their ${ }^{1} \mathrm{H}-\mathrm{NMR}$ spectra were certificated. Rat liver microsomes, solutions of NADPH coenzymes A and B, were all purchased from $\mathrm{BD}$ Biosciences (Bedford, MA, USA) and stored at $-70^{\circ} \mathrm{C}$. Fresh rat blood was extracted from a healthy male Sprague-Dawley rat (Bio LASCO Co., Taiwan) and centrifuged to obtain plasma.

\subsection{Analytical Equipment}

The purities of the synthesized INER-1577s, and their molecular fragmentation mechanisms and biotransformation behavior in rat liver microsomes and rat plasma, were analyzed using an HPLC system (Agilent 1100/1200 series, Palo Alto, CA, USA) with an online degasser, binary pump, autosampler, and diode array detector (DAD). Data were acquired and processed using Agilent ChemStation 10.02 software. The HPLC system was coupled with a tandem mass spectrometer (4000QTRAP, AB Sciex, Concord, ON, Canada, using Analyst 1.6.2 software via a splitter (10:1). The mass spectrometer was equipped with an electrospray ionization source, and a triple quadrupole linear ion trap mass detector and was operated in positive-ion detection mode. 


\subsection{Validation of HPLC-MS/MS Analytical Method for Determination of INER-1577 \#3}

The HPLC-MS/MS analytical method was set up and validated for the determination of INER-1577 \#3 and was then employed to determine the purities, molecular fragmentation mechanisms and bio-reactivity of the INER-1577s in various biosystems. The linearity of the calibration curve, dynamic range, precision, recovery, and reproducibility of the LC-MS method and the frozen/thawed stability were assessed to confirm the suitability of the method. The LC-MS method was employed to determine the purities of INER-1577 \#1 - \#4 and the biotrans formation rates of INER-1577 \#3 and \#4. The respective fragmentation mechanisms of INER-1577 \#1 - \#4 and the identification of their metabolites were proposed according to their tandem mass spectra.

\subsection{Procedures Used for Biotransformation Studies of INER-1577s}

The studied INER-1577 standards were separately dissolved in DMSO (1 $\mathrm{mg} \cdot \mathrm{mL}^{-1}$ ) and mixed with bio-reaction systems (rat liver microsomes and rat plasma) for several time intervals (up to $4 \mathrm{~h}$ ) before the bio-reaction was quenched by the addition of methanol (1:1 volume), followed by mixing and centrifugation $\left(6000 \mathrm{rpm}\right.$ at $4^{\circ} \mathrm{C}$ for $\left.10 \mathrm{~min}\right)$. The resulting supernatant was filtered through a $0.22-\mu \mathrm{m}$ PVDF disk filter and analyzed via HPLC-MS/MS. Details of the conditions used for incubation of the tested compounds with rat liver microsomes and rat plasma are provided in [22], and the directions followed are given in [23] (microsomes) and [24] (plasma).

\section{Results and Discussion}

\subsection{Development and Validation of LC-MS Analytical Method for Determination of INER-1577 \#3}

To determine the synthesis qualities, and bio-reaction rates of the INER-1577s and identify their derivatives, reverse phase HPLC-MS/MS was initially set up for the determination of INER-157 7\#3. This procedure was conducted on a C18 column (Agilent ZORBAX Eclipse XDB-C18, $4.6 \mathrm{~mm} \times 50 \mathrm{~mm}, 1.8 \mu \mathrm{m}$ ) with an aqueous ammonium acetate $(10 \mathrm{mM}) /$ methanolic mobile phase in programmed elution. Quantification of the chemicals was performed using the DAD $(250 \mathrm{~nm})$ and multiple reaction monitoring (MRM) of parent-product ion $\mathrm{m} / \mathrm{z}$ pairs with a mass spectrometer detector (MSD). Chromatograms were recorded up to 17 min with turnaround time of 36 min per injection. The instrumental parameters are listed in Table 1 . The chromatogram of INER-1577 \#3 is shown in Figure $2(a)$. and the retention time $\left(t_{R}, \min \right)$, theoretical plate number $(N)$, resolution factor, selectivity factor and tailing factor were $11.8 \pm 0.15,18,400,1.75,1.06$, and 1.15 , respectively. The chromatographic purity was $99.55 \%$ based on the ratio of its peak area to those of all component peaks (DAD detection, except for the system and background peaks). The dynamic range was $0.5-100 \mathrm{mg} \cdot \mathrm{L}^{-1}$ with 
Table 1. Analytical parameters of LC-MS/MS used for the study of the fragmentation mechanism and metabolism of the INER-1577 series of HDACi PET imaging agents.

\begin{tabular}{|c|c|}
\hline \multicolumn{2}{|r|}{ HPLC } \\
\hline Stationary phase & Agilent, Eclipse XDB-C18, $4.6 \mathrm{~mm} \times 50 \mathrm{~mm}, 1.8 \mu \mathrm{m}$ \\
\hline Injection volume, $\mu \mathrm{L}$ & 3 \\
\hline Detector & $\mathrm{DAD}$ at $250 \mathrm{~nm}$ \\
\hline Mobile phase & $\begin{array}{l}\text { A: } 10 \mathrm{mM} \mathrm{NH}_{4} \mathrm{Ac}_{(\mathrm{aq})} 90 \% / \mathrm{MeOH} 10 \% \\
\text { B: } 10 \mathrm{mM} \mathrm{NH}_{4} \mathrm{Ac}_{(\mathrm{aq})} 20 \% / \mathrm{MeOH} 80 \% \\
\text { Degassed in ultrasonic bath for } 20 \mathrm{~min}\end{array}$ \\
\hline Gradient program & $\begin{array}{l}0 \text { - } 3 \text { min } 50 \% \text { B, isocratic } \\
3 \text { - } 12 \text { min up to } 100 \% \text { B, held for } 5 \mathrm{~min} \\
17 \text { - } 23 \text { min down to } 5 \% \text { B, held for } 5 \text { min to wash out salts } \\
\text { from biomatrices } \\
28 \text { - } 31 \text { min up to } 50 \% \mathrm{~B} \text {, held for } 4 \text { min to re-condition column }\end{array}$ \\
\hline Flow rate, $\mathrm{mL} \cdot \mathrm{min}^{-1}$ & 0.5 \\
\hline \multicolumn{2}{|r|}{ Electrospray mass spectrometry } \\
\hline Temperature $\left({ }^{\circ} \mathrm{C}\right)$ & 400 \\
\hline Detector polarity & Positive ion \\
\hline Scanning mass range & $100-1000$ \\
\hline Resolution, Q1 and Q3 & Unit \\
\hline Nebulizer gas (p.s.i.) & 40 \\
\hline Curtain gas (p.s.i.) & 10 \\
\hline Collision gas (p.s.i.) & Medium or high for EMS and EPI scan modes ${ }^{\mathrm{a}}$, respectively \\
\hline Ion spray voltage $(\mathrm{V})$ & 5000 \\
\hline Declustering potential (V) & 40 \\
\hline
\end{tabular}

${ }^{a}$ Scan mode: EMS-enhanced mass spectrometry; EPI-enhanced product ion.

a slope of $9.2\left(\mathrm{R}^{2}>0.999, \mathrm{DAD}\right)$ and $5-500 \mu \mathrm{g} \cdot \mathrm{L}^{-1}\left(\mathrm{R}^{2}>0.995, \mathrm{MSD}\right.$ for MRM pair $392 \rightarrow 162$ and $392 \rightarrow 347$, the inset of Figure 2(a)). The accuracies (averages of triplicate measurements at low, intermediate, and high concentrations of $2.5,37.5$, and $75 \mathrm{mg} \cdot \mathrm{L}^{-1}$ ) were $95.6 \%-101.6 \%$. Determination of the intra-day and inter-day precisions of triplicate measurements at various concentrations indicated that the biases were less than $2.2 \%$ and $2.8 \%$, respectively.

For validation of the bio-analytical method solutions of INER-1577 \#3 with nominated concentrations were spiked in biomatrices (including liver microsomes, NADPH coenzymes), which were vortexed well, and the analytes were immediately separated to determine a calibration curve for INER-1577 \#3 in bio-samples. The results showed that the dynamic range was $2-250 \mathrm{mg} \cdot \mathrm{L}^{-1}$ with a slope of $9.4\left(\mathrm{R}^{2}>0.998\right.$, DAD detection $)$ and $0.5-100 \mathrm{mg} \cdot \mathrm{L}^{-1}\left(\mathrm{R}^{2}>0.984\right.$, MRM detection). The extraction recovery was estimated to be approximately $50 \%$. The accuracies of the determination of INER-1577 \#3 in biomatrices at concentrations of 10,100 , and $250 \mathrm{mg} \cdot \mathrm{L}^{-1}$ were shown to be $141.5 \%, 96.9 \%$, and 93.6\%, respectively. The intra-day and inter-day precisions for the analysis of INER-1577 \#3 in biomatrices showed that all relative standard deviations (RSD) were between $0.3 \%$ and $1.2 \%$. To confirm the stability of INER-1577 \#3 after being 
frozen at $-20^{\circ} \mathrm{C}$ and then thawed at $0^{\circ} \mathrm{C}$ for one and three cycles, sample solutions $\left(10,100\right.$, and $\left.250 \mathrm{mg} \cdot \mathrm{mL}^{-1}\right)$ were analyzed in triplicate. The average accuracies (\%) were $141.7 \%, 97.0 \%$, and $94.0 \%$ (one cycle) and $141.6 \%, 97.1 \%, 93.6 \%$ (three cycles), respectively. No derivative was found to appear. Therefore,

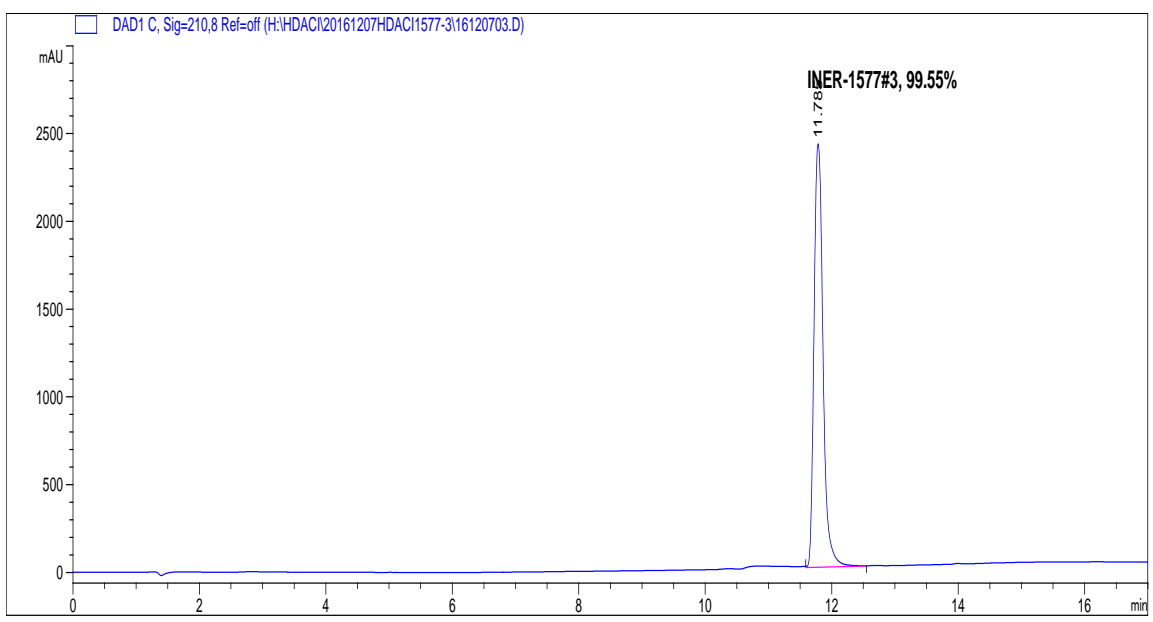

(a)

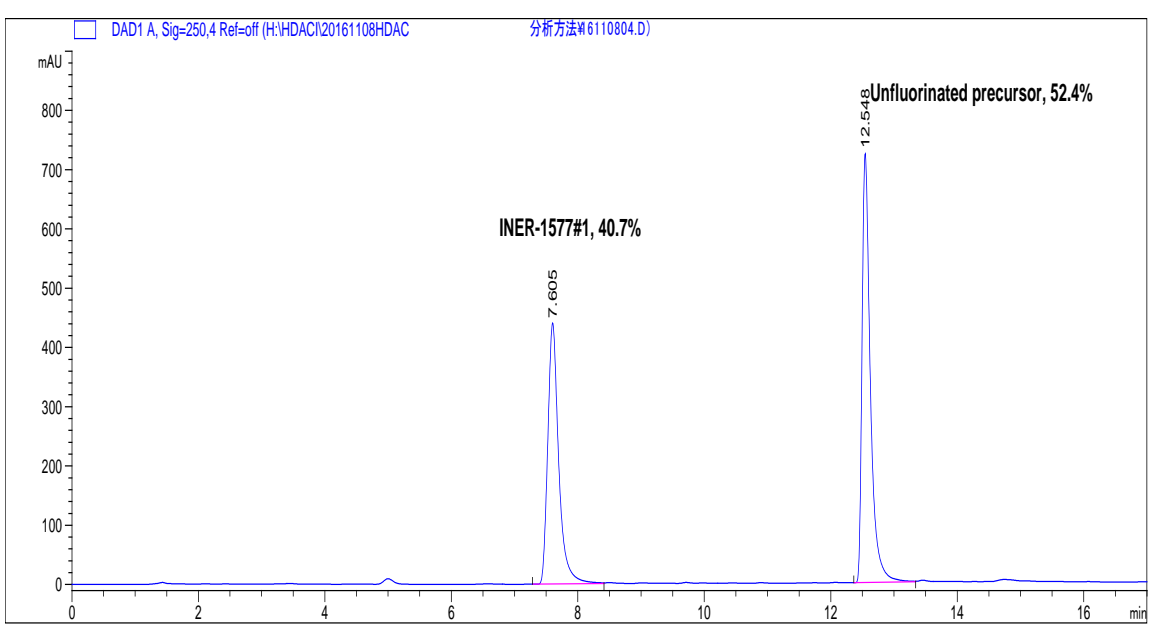

(b)

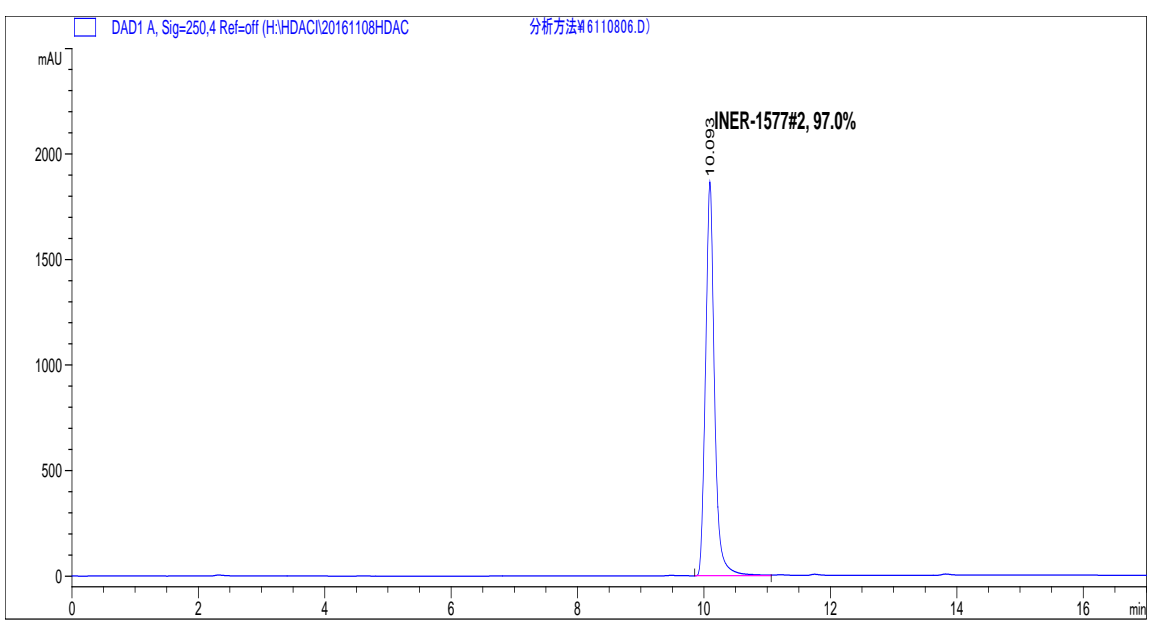

(c) 


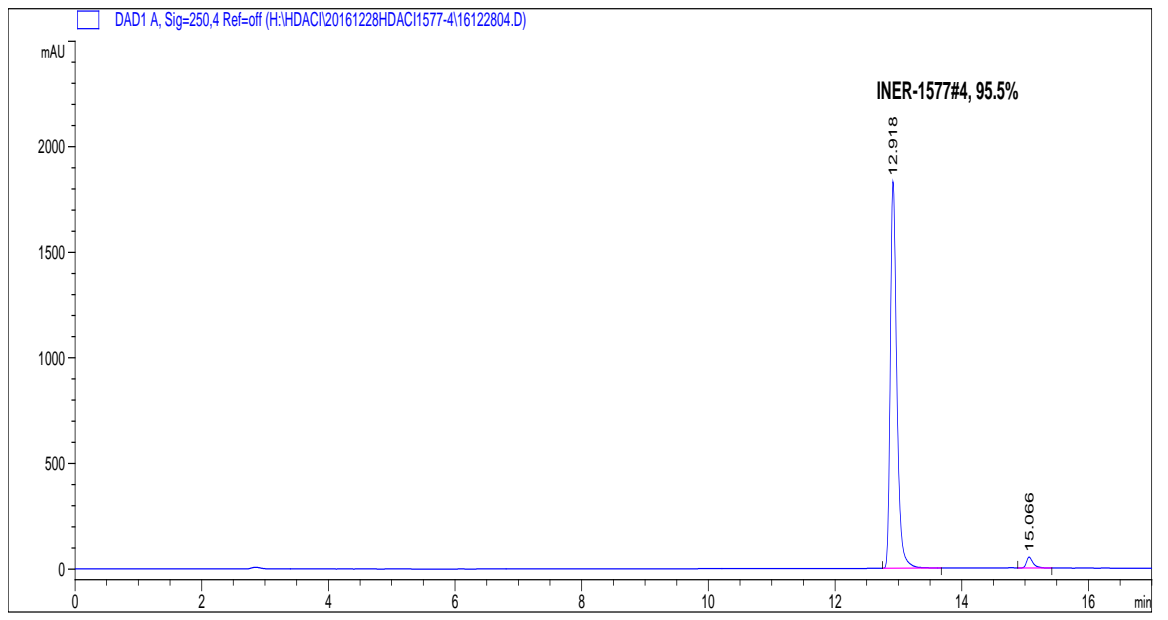

Figure 2. HPLC-UV chromatograms of INER-1577s: (a) \#3, (b) \#1, (c) \#2, and (d) \#4.

INER-1577 \#3 would not be obviously spoiled after being frozen, stored, and then thawed for analysis. The analytical method used for the determination of INER \#3 was thus suitable for the quantitation of bio-matrix samples.

\subsection{Application to Determine the Purities of INER-1577 Analogs}

Before the characteristics of a chemical are studied, it is important to check its quality. The analytical method used for INER-1577 \#3 was also employed to determine the purities of \#1, \#2, and \#4. The chromatograms of the analogs are shown in Figures 2(b)-(d). The purity of INER-1577 \#1 was $40.7 \%$ with a retention time $\left(\mathrm{t}_{\mathrm{R}}\right) 7.6 \mathrm{~min}(\mathrm{~m} / \mathrm{z}=365)$, and the major impurity in the material was its non-fluorinated precursor $(\mathrm{m} / \mathrm{z}=347)$, of which the content was $52.4 \%\left(t_{R}=\right.$ $12.55 \mathrm{~min})$. The efficiency of the fluorination reaction used to prepare INER-1577 \#1 was thus unsatisfactory. For INER-1577 \#2 analogue, the chromatographic purity was $97.0 \%(\mathrm{~m} / \mathrm{z}=364)$ and $t_{R}$ was $10.1 \mathrm{~min}$, accompanied by six insignificant impurities. For INER-1577 \#4, $\mathrm{t}_{\mathrm{R}}$ was $12.9 \mathrm{~min}$ and the chromatographic purity was $95.5 \%(\mathrm{~m} / \mathrm{z}=482)$ with seven detectable minor impurities.

\subsection{Fragmentation Mechanisms of INER-1577s}

To determine the chemical bonding characters and compile a basic data bank of the mass spectra of the HDACi analogs, which is helpful for the identification of derivatives and to understand structural weaknesses, their fragmentation mechanisms were studied using tandem mass spectra. The fragment structures and fragmentation routes with the corresponding $\mathrm{m} / \mathrm{z}$ values of the product ions are outlined in Figures 3(a)-(d) for INER-1577 \#1 - \#4, in sequence. From an overview of the schemes, it is obvious that the labile bonds included the phenyl and alkyl C-F, benzamide, and tertiary amine bonds. Secondly, a few equivalent fragment ions were detected for all of INER-1577 \#1 - \#3, which arose from the loss of $\mathrm{HN}\left(\mathrm{CH}_{3}\right)_{2}(\Delta \mathrm{m} / \mathrm{z}=-45), \mathrm{CO}(\Delta \mathrm{m} / \mathrm{z}=-28),\left(\mathrm{CH}_{3}\right)_{2} \mathrm{NCH}(\Delta \mathrm{m} / \mathrm{z}=-57)$, and $\mathrm{F}$ (coupled with $\mathrm{H}, \Delta \mathrm{m} / \mathrm{z}=-18$ ) groups. This information is also beneficial for potential molecular modification and prediction of the characteristics of the 
products.

\subsection{Identification of Metabolites of INER-1577s in Biosystems}

Because the structures of the four INER-1577 analogs are similar, the types of chemical bond in INER-1577 \#3 and \#4 could represent those in \#1 and \#2. The biochemical behavior of the compounds was therefore studied by incubating \#3 and \#4 in rat liver microsomes and/or rat plasma. A liver microsome protocol is often used to study the metabolic pathways of xenobiotics and the catalysis of

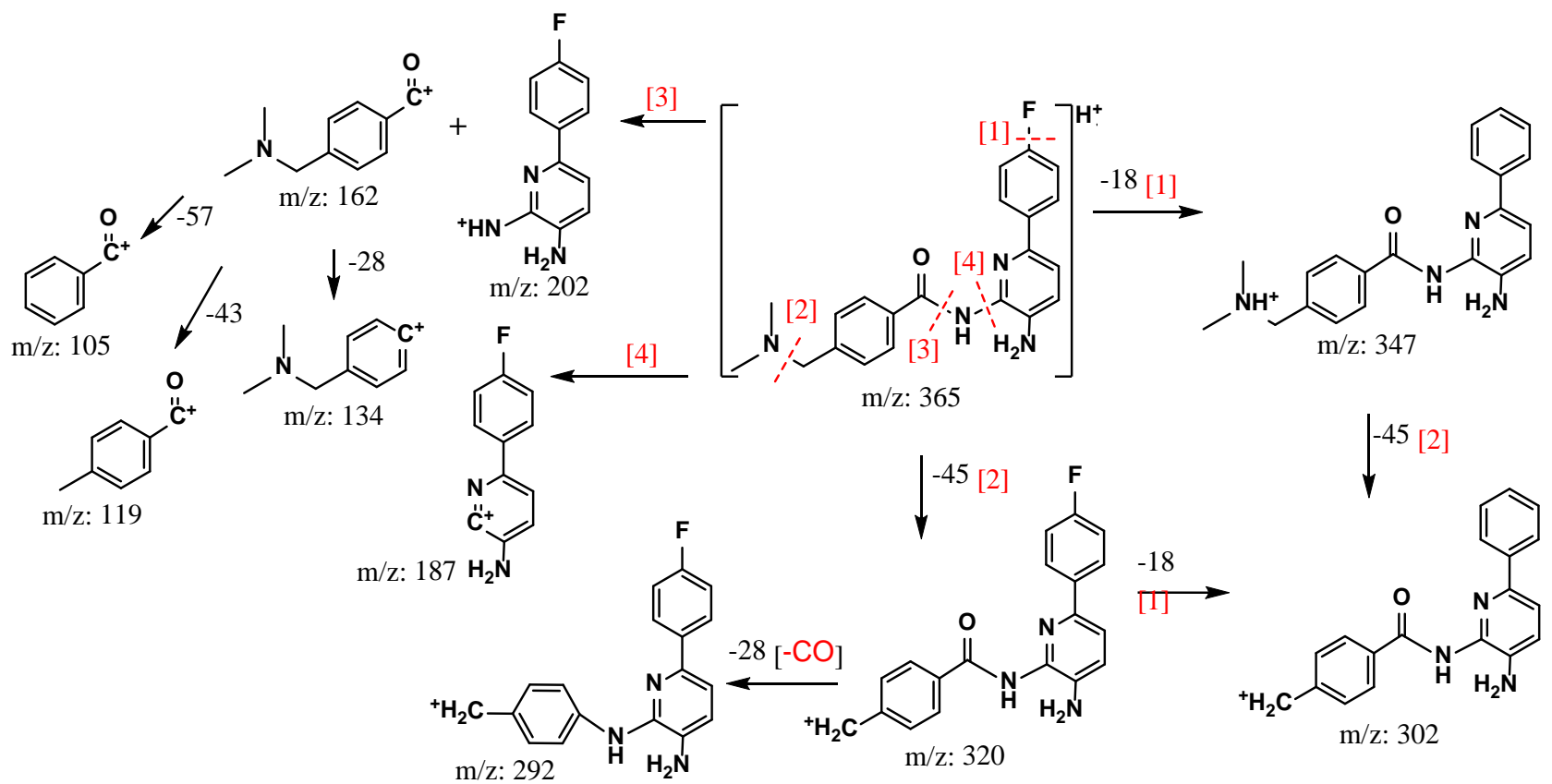

(a)<smiles>Cc1ccc([C+]=O)cc1</smiles>

(b) 


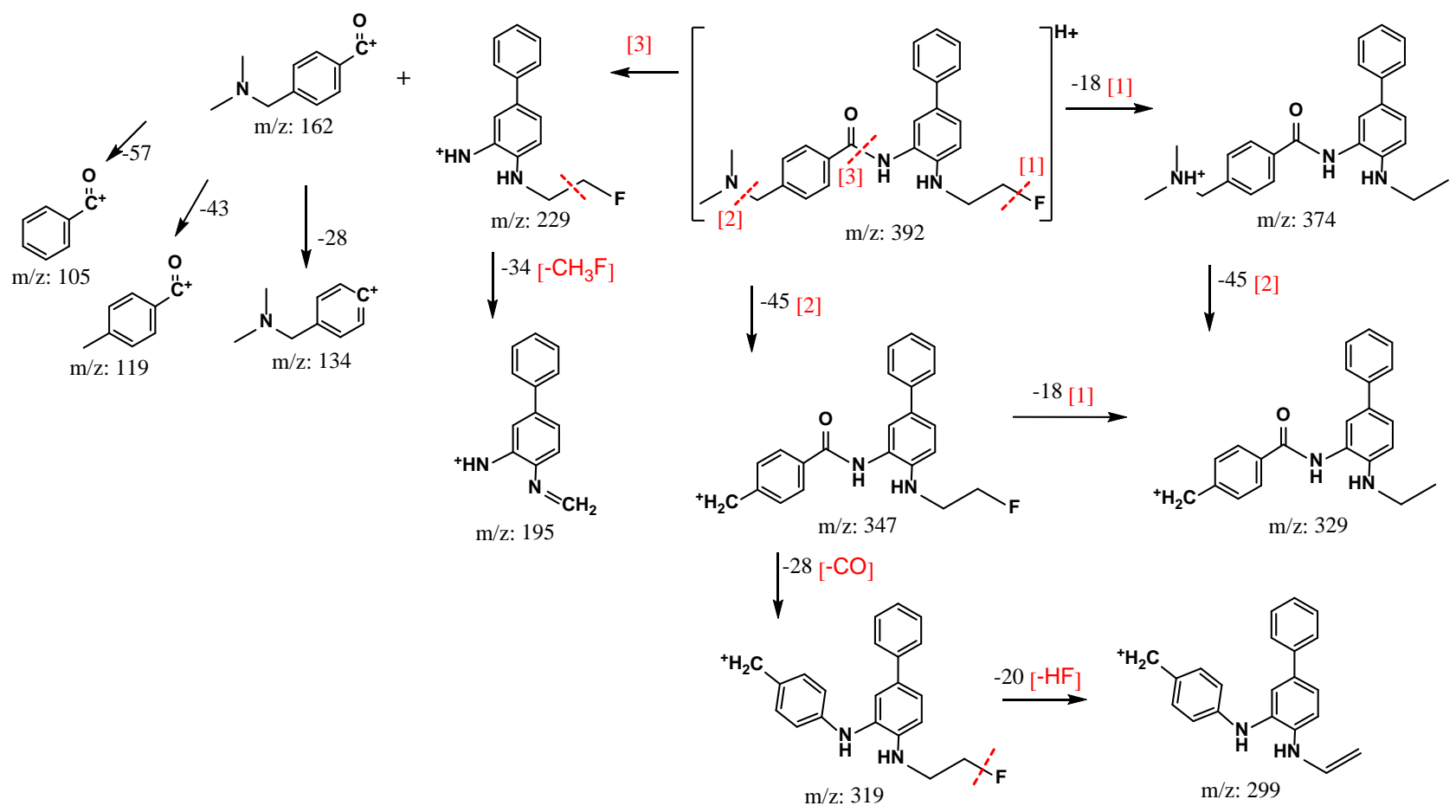

(c)

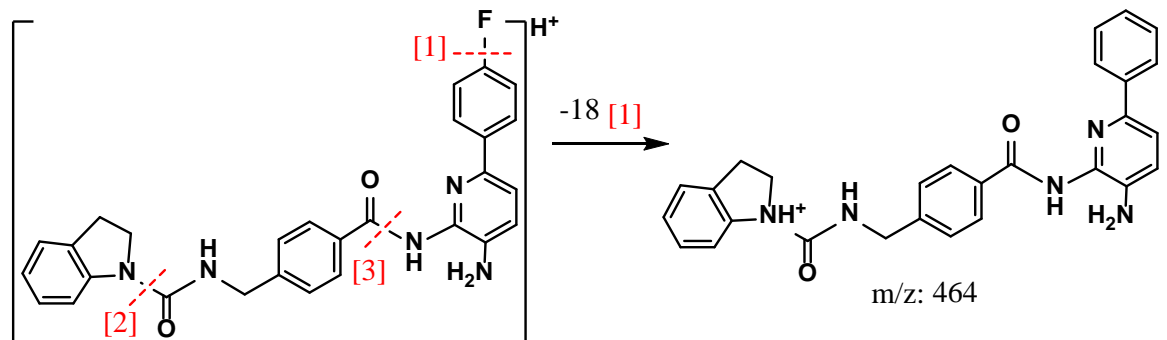
m/z: 482<smiles>COc1ccc(CNC(=O)N2CCc3ccccc32)cc1</smiles>
$\mathrm{m} / \mathrm{z}: 279$

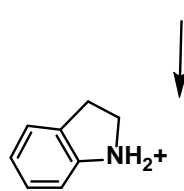

m/z: 120<smiles>O=C=NCc1ccc([C+]=O)cc1</smiles>
m/z: 160

(d)

Figure 3. Molecular fragmentation schemes of INER-1577s: (a) \#1, (b) \#2, (c) \#3, and (d) \#4.

phase I and II reactions [23]. In the study, no endogenic substances such as sulfate, glucose, amines, or amino acids were supplied. Those are required by phase II reactions, and conjugated products could therefore not be detected in the system. In general, phase II conjugated metabolites are non-bioactive. On the other hand, the tested materials are administered via intravenous injection (iv) and are 
delivered to the organs and CNS by the body's circulatory system, and might be metabolized in the blood before they arrive at the CNS to bind with the specified sites, as was originally intended. Therefore, a study of its metabolic pathway and metabolization rate in plasma is pivotal for the appropriate use of a drug [24].

Firstly, INER-1577 \#3 was biotransformed in a rat liver microsomes/NADPH coenzyme system for a specified period, and then its abundance was monitored via its chromatographic peak area (DAD $250 \mathrm{~nm}$ ) over a period time after the incubated solutions were pretreated. The chromatographic separation of INER-1577 \#3 and its metabolites in liver microsomes is shown in Figure 4(a) which demonstrates that one major metabolite $\left(\mathrm{M} 4_{\# 3}, \mathrm{t}_{\mathrm{R}}=10.36 \mathrm{~min}\right)$ and three minor metabolites $\left(\mathrm{M1}_{* 3}-\mathrm{M}_{* 3}, \mathrm{t}_{\mathrm{R}}=3.92,6.40\right.$, and $7.01 \mathrm{~min}$, respectively) were produced. The inset shows the trends in the abundance of INER-1577 \#3 and $\mathrm{M} 4_{\# 3}$. The biotransformation of INER-1577 \#3 in liver microsomes was obvious during the first half-hour, after which less than $30 \%$ of the compound remained; after $90 \mathrm{~min}$ it was entirely consumed. The $\mathrm{m} / \mathrm{z}$ data for the tandem mass spectra and the structures of the product ions and metabolites of $\mathrm{M} 1_{\# 3}$ to $\mathrm{M} 4_{\# 3}$ are proposed in Figures 5(a)-(d). Because the $\mathrm{m} / \mathrm{z}$ value of the parent ion of $\mathrm{M} 4_{\# 3}$ was $408(\Delta \mathrm{m} / \mathrm{z}=+16$ with respect toINER-1577 $\# 3$ as a result of oxidation), and the $\mathrm{m} / \mathrm{z}$ value of the fragment ions were 347,329 , and 195 , it was believed to be a product of hydroxylation of the methylamino group. The $\mathrm{m} / \mathrm{z}$ data for the fragment ions and the mechanism support the identity of metabolites $\mathrm{M} 1_{\# 3}-\mathrm{M} 4_{\# 3}$.

Secondly, INER-1577 \#3 was incubated with plasma for various durations and its abundance was determined. The resulting chromatograms (DAD detection, Figure $4(\mathrm{~b}))$ indicate that one metabolite, namely, $M 5_{\# 3}$ was produced $\left(t_{R}=\right.$ $11.87 \mathrm{~min}, \mathrm{MS}^{1} \mathrm{~m} / \mathrm{z}=229$ and $\mathrm{MS}^{2} \mathrm{~m} / \mathrm{z}=212,209,195$, and 183). The abundances of INER-1577 \#3 and $M 5_{\# 3}$ are plotted against time in the inset of Figure 4(b) which shows that INER-1577 \#3 was metabolized to an extent approaching consumption ( $7.1 \%$ remaining) after $1 \mathrm{~h}$. The product that was found was formed by the hydrolysis of the benzamide group to give a diaminobiphenyl compound, and meanwhile ahydroxyl group had replaced the fluorine atom. The other hydrolysis product of INER-1577 \#3, namely, $\mathrm{M} 6_{\# 3}$, or 4-((dimethylamino)methyl)benzoic acid, was found using MSD (extracted ion count, XIC, $\mathrm{m} / \mathrm{z}=180$ ) at $\mathrm{t}_{\mathrm{R}}=1.77 \mathrm{~min}$ with $\mathrm{MS}^{2} \mathrm{~m} / \mathrm{z}=162$ and 134 . The mass spectral data and structures of $\mathrm{M} 5_{\# 3}$ and $\mathrm{M} 6_{\# 3}$ are shown in Figure 5(e) and Figure 5(f).

Lastly, INER-1577 \#4 was incubated with rat liver microsomes for up to $2 \mathrm{~h}$. The chromatograms and reaction trend of metabolized solutions of INER-1577 \#4 are shown in Figure $4(\mathrm{c})$ and its inset. Two significant metabolites $\left(\mathrm{M} 1_{* 4}\right.$, and $\left.M 3_{* 4}\right)$ and one minor metabolite $\left(M 2_{* 4}\right)$ were found $\left(t_{R}=2.7,6.55,9.75\right.$ min for $\mathrm{M1}_{* 4}, \mathrm{M}_{4_{4}}$, and $\mathrm{M}_{* 44}$, respectively). The tandem mass spectral data and proposed fragment ion structures used to indicate identity of metabolites $\mathrm{M} 1_{* 4}-\mathrm{M} 3_{\sharp 4}$ are shown in Figures 5(g)-(i). 


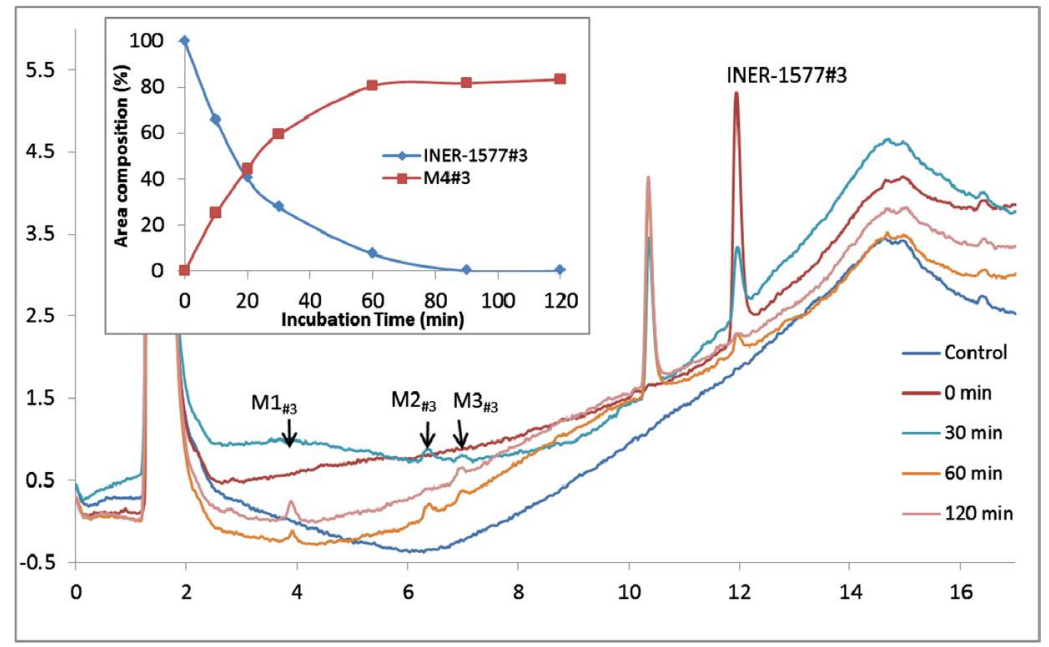

(a)

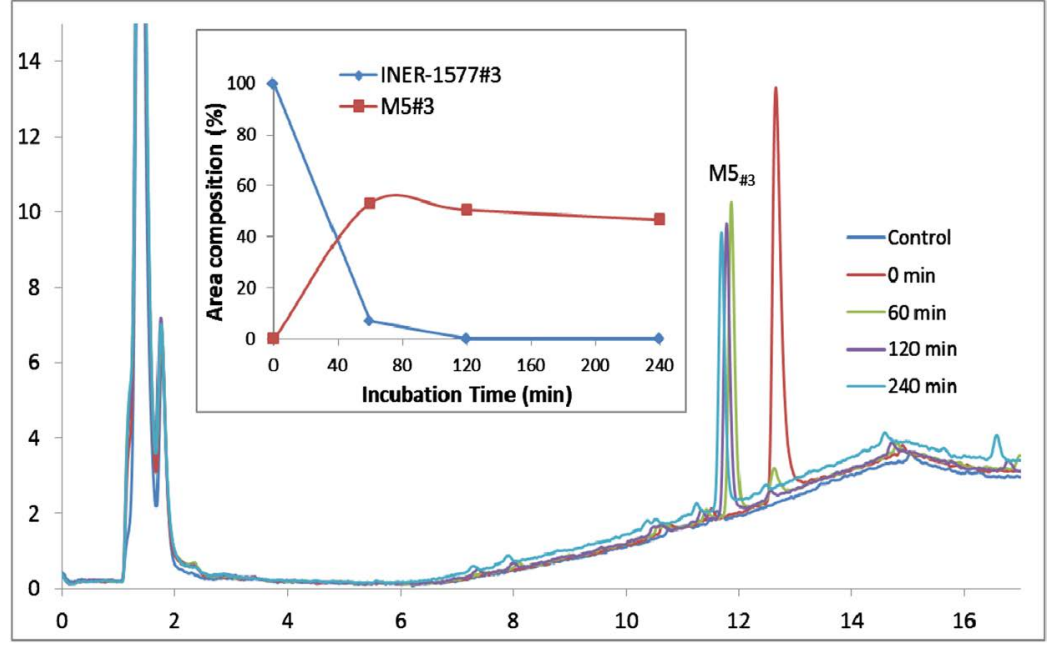

(b)

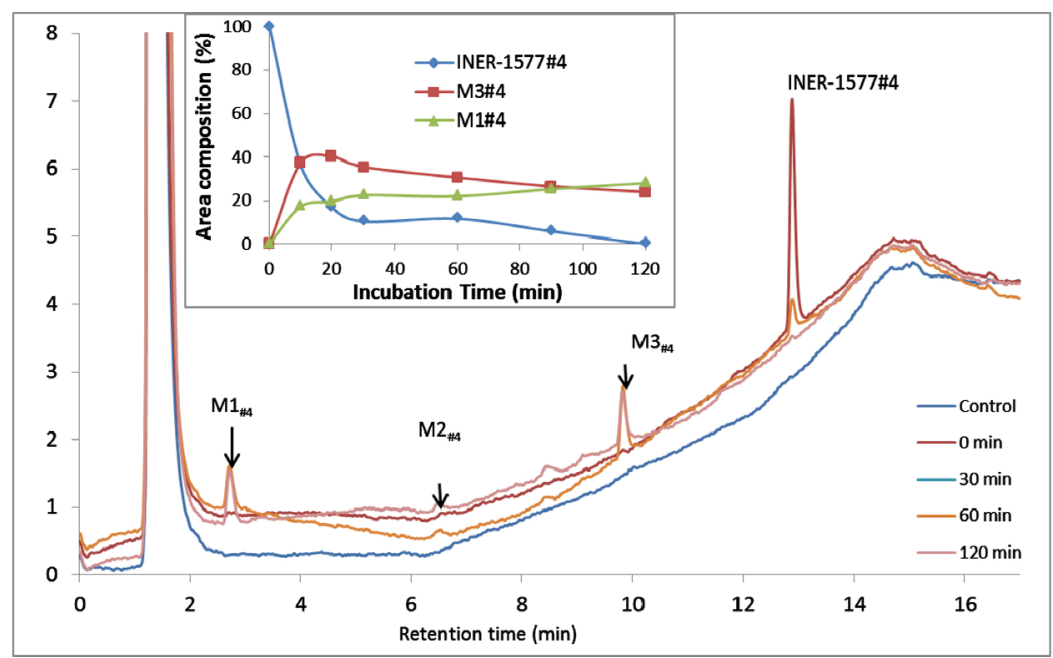

Figure 4. Chromatograms of INER-1577s incubated with biosystems: (a) INER-1577 \#3/liver microsomes; (b) INER-1577 \#3/plasma; and (c) INER-1577 \#4/liver microsomes. The inset showed the trends in the abundance of the parent compounds and major metabolites during the reactions. 


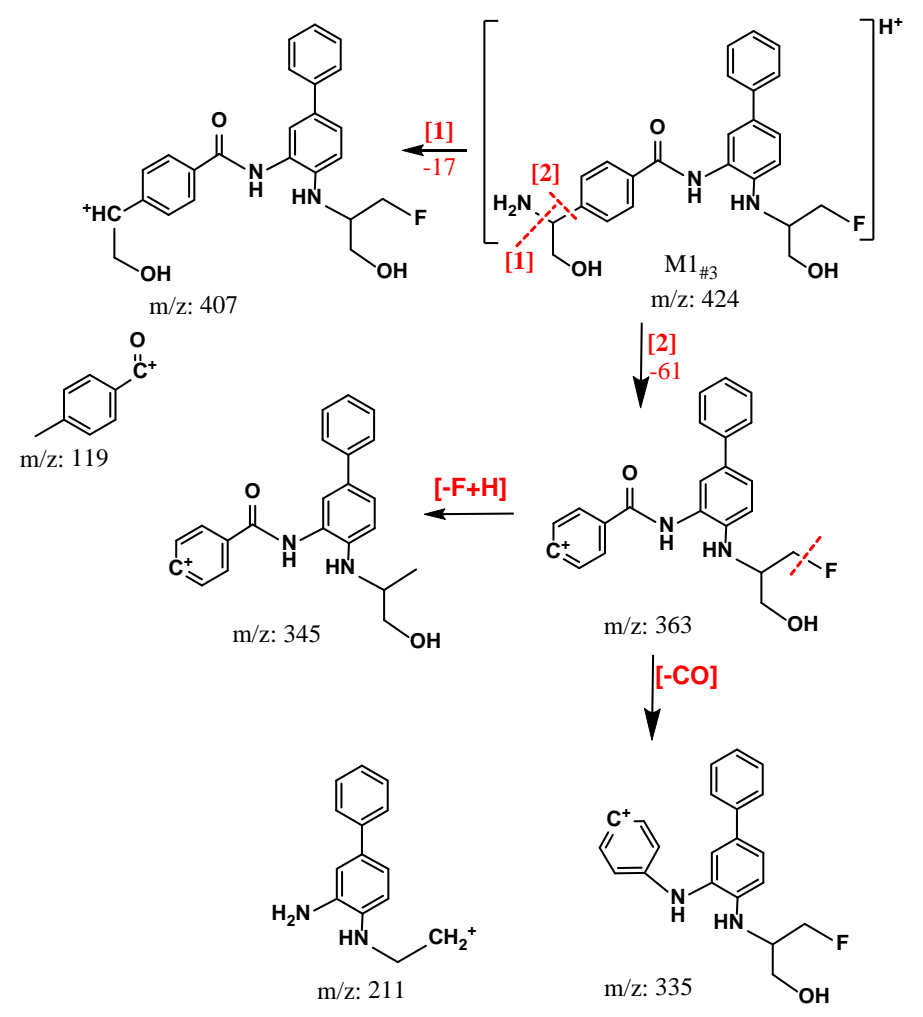

(a)

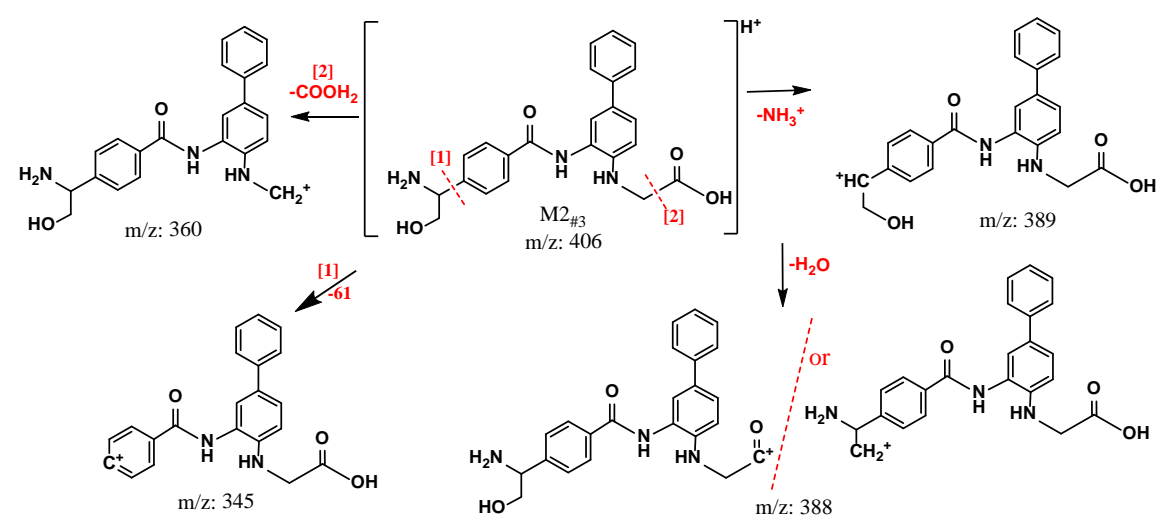

(b)

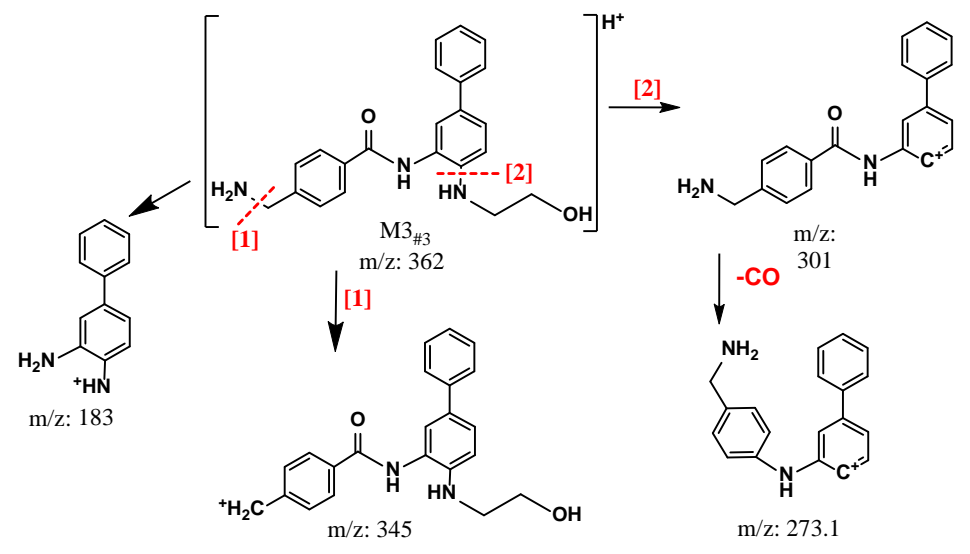

(c) 


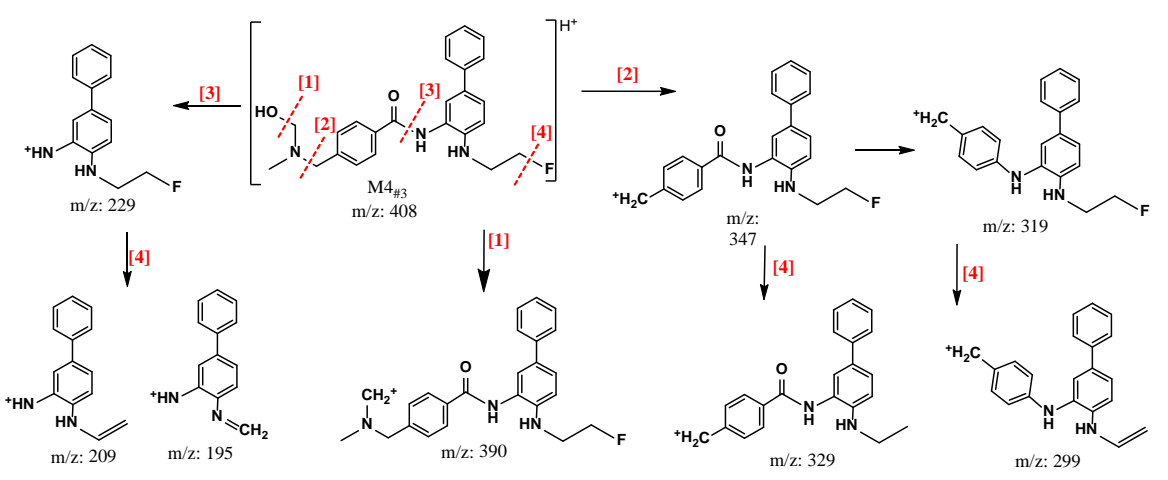

(d)

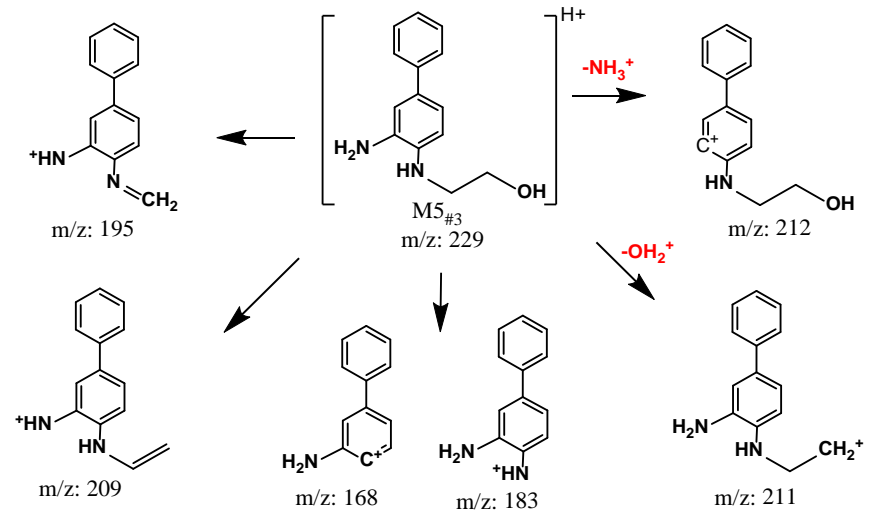

(e)

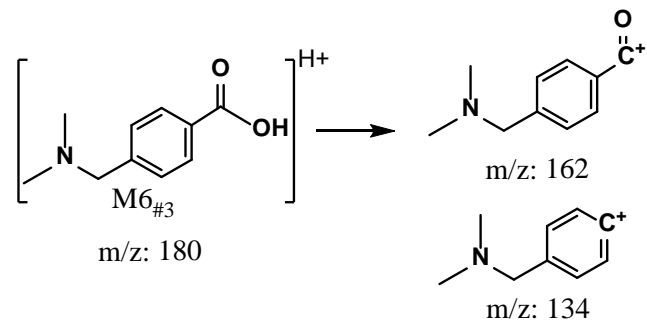

(f)

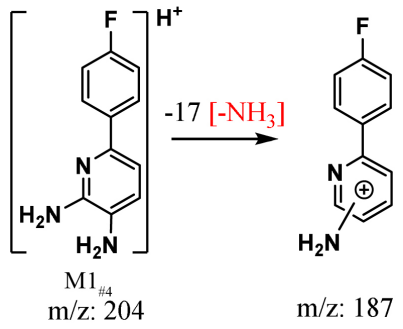

(g)
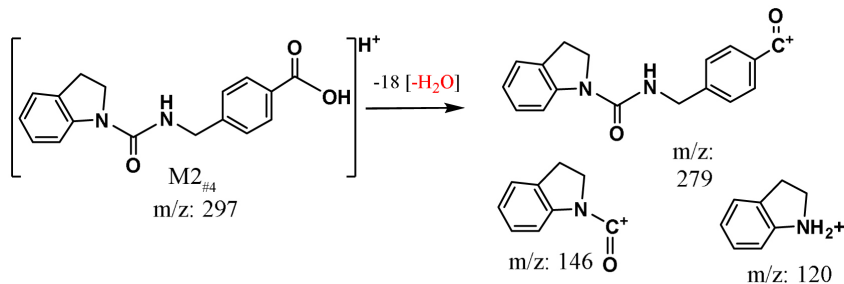

(h) 


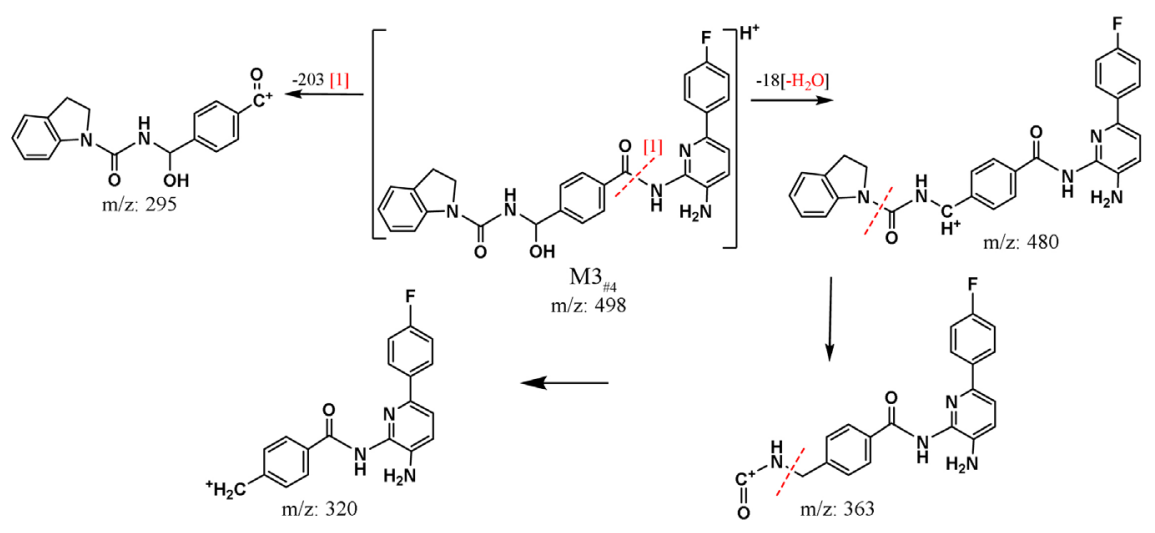

(i)

Figure 5. Fragmentation mass spectra data and structures of metabolites of INER-1577 \#3 and \#4.

The mechanisms of the metabolism of INER-1577 \#3 in liver microsomes and plasma, and \#4 in liver microsomes are outlined in Figure 6(a) and Figure 6(b), respectively. The biotransformation of INER-1577 \#3 by liver microsomes was diverse including demethylation or hydroxylation of the dimethylamino group, methylene hydroxylation of the benzylamino and fluoroethylamino groups, the replacement of the fluorine atom by a hydroxyl group, and carboxylation of the fluorine-bearing carbon atom. On the other hand, for INER-1577 \#3 in plasma only hydrolysis of the benzamide group and, at the same time, the substitution of a hydroxyl group for the fluorine atom whereas observed. Fluorine atom might be removed from INER-1577 \#3 after it had been biotransformed, and its partial metabolites were thus untraceable by PET graph. The metabolism of INER-1577 \#4 by liver microsomes proceeded via two routes: hydrolysis of the benzamide group to give a pair of products, namely, benzoic acid and diaminopyridylmoieties, and hydroxylation of the methylene carbon of the benzyl-amino group. The fluorine atom on the phenyl group of INER-1577 \#4 was not removed from the ligand, which thus remained radio-traceable. Therefore, the bio-stability of F-C bonds is less for alkyl-F bonds than for phenyl-F bonds. Because the indolino group of INER-1577 \#4 restricted reactions at the tertiary amine group, its biotransformations were simpler than those of INER-1577 \#3. Moreover, the potential metabolites of INER-1577 \#3 and \#4 produced in the liver and plasma were more hydrophilic and it was thus difficult for them to cross the blood-brain barrier (BBB) into the CNS for imaging. Because HDACs are zinc-centered metallo-enzymes [25], and the benzamide groups of the INER-1577 analogs compete with amino acids for coordination with zinc atoms [26], the function of the analogs is terminated after hydrolysis of the benzamide groups in the liver or blood. Although the researches indicated that benzamide derivatives showed anticancer activities under phase II clinical trials based on HDAC inhibition, there is no information available on its microsomal stability and metabolism for mocetinostat (MGCD0103) and no metabolites could be detected after incubation of MS-275 in human liver microsomes for entinostat (MS-275) [27]. 

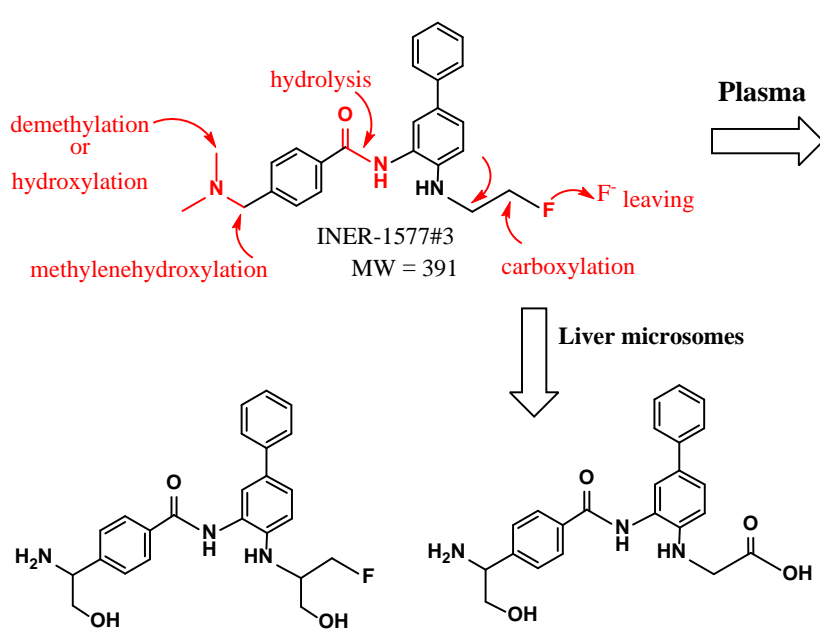

4-(1-amino-2-hydroxyethyl)- $N_{-}$(4-((1fluoro-3-hydroxypropan-2-yl)amino) -[1,1'-biphenyl]-3-yl)benzamide Chemical Formula: $\mathrm{C}_{24} \mathrm{H}_{26} \mathrm{FN}_{3} \mathrm{O}_{3}$ MW $=423$

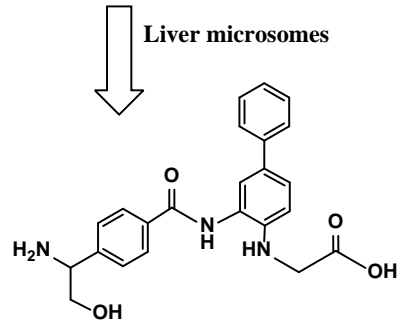

(3-(4-(1-amino-2-hydroxyethyl) benzamido)-[1,1'-biphenyl]-4-yl)glycine Chemical Formula: $\mathrm{C}_{23} \mathrm{H}_{23} \mathrm{~N}_{3} \mathrm{O}_{4}$<smiles>Nc1cc(-c2ccccc2)ccc1CCO</smiles>

2-((3-amino-[1,1'-biphenyl]

-4-yl)amino)ethan-1-ol Chemical Formula: $\mathrm{C}_{14} \mathrm{H}_{16} \mathrm{~N}_{2} \mathrm{O}$ $\mathrm{MW}=228$

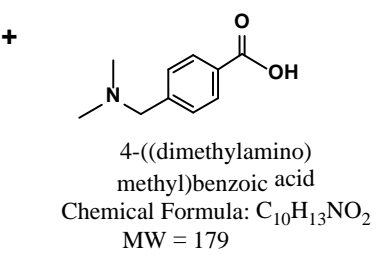

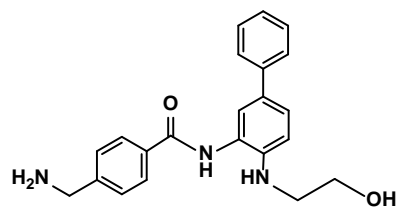

4-(aminomethyl)- $N_{-}$(4-((2-hydroxyethyl) amino)-[1,1'-biphenyl]-3-yl)benzamide Chemical Formula: $\mathrm{C}_{22} \mathrm{H}_{23} \mathrm{~N}_{3} \mathrm{O}_{2}$ $\mathrm{MW}=361$

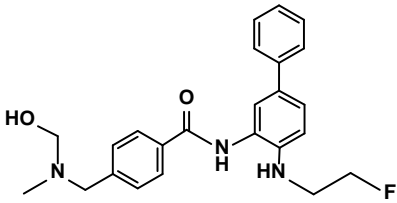

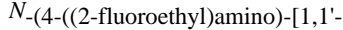
biphenyl]-3-yl)-4-(((hydroxymethyl) (methyl)amino)methyl)benzamide Chemical Formula: $\mathrm{C}_{24} \mathrm{H}_{26} \mathrm{FN}_{3} \mathrm{O}_{2}$

(a)

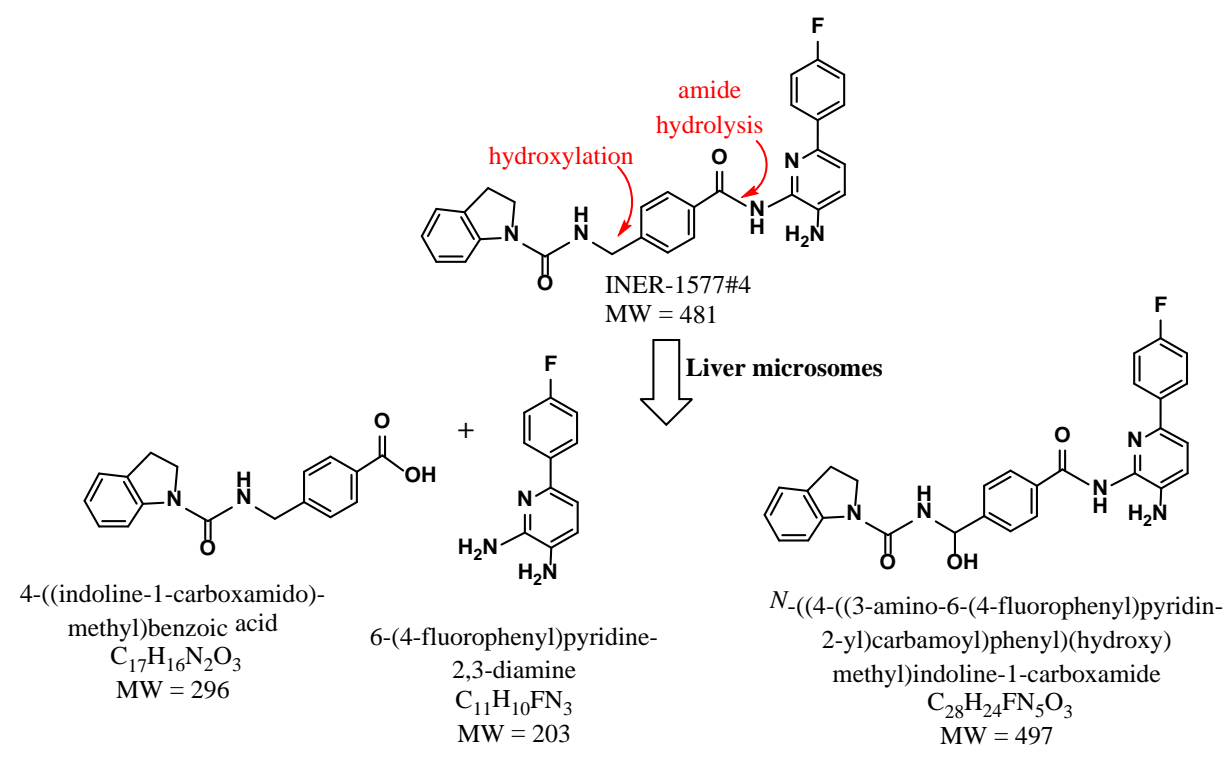

(b)

Figure 6. Metabolic pathways of INER-1577s in biomatrices: (a) INER-1577 \#3 in liver microsomes and plasma; (b) INER-1577 \#4 in liver microsomes.

\section{Conclusion}

According to the study results, the reactive chemical bonds and biochemical characteristics of the INER-1577 analogs were well defined. It is suggested that PET imaging should be performed immediately after the ligand reaches distribution equilibrium in the CNS and end within $1 \mathrm{~h}$ after i.v. administration. The ligands are inactivated after biohydrolysis of the benzamide groups of INER-1577 \#3 and \#4 to give smaller molecules, and hydroxylation to give more hydrophilic metabolites could promote the excretion of the ligand via urine. Although the 
radioactive nuclide ${ }^{18} \mathrm{~F}$ is removed from the INER-1577 \#3 ligand after metabolism and is transported around the body, its half life is short $\left(109 \mathrm{~min}\right.$, with ${ }^{18} \mathrm{O}$ as its daughter nuclide) and it is easily excreted via urine. Therefore, the bio-safety of the INER-1577s has been confirmed.

\section{Acknowledgements}

The study was supported by a grant from the Atomic Energy Council, Taiwan (Grant No. A-IE-02030201).

\section{References}

[1] Small, G.W., Siddarth, P., Kepe, V., Ercoli, L.M., Burggren, A.C., Bookheimer, S.Y., Miller, K.J., Kim, J., Lavretsky, H., Huang, S.C. and Barrio, J.R. (2012) Prediction of Cognitive Decline by Positron Emission Tomography of Brain Amyloid and Tau. Archives of Neurology, 69, 215-222.

https://doi.org/10.1001/archneurol.2011.559

[2] Lu, F.M. and Yuan, Z. (2015) PET/SPECT Molecular Imaging in Clinical Neuroscience: Recent Advances in the Investigation of CNS Diseases. Quantitative Imaging in Medicine and Surgery, 5, 433-447.

[3] Asbury, C. (2011) Brain Imaging Technologies and Their Applications in Neuroscience. The DANA Foundation.

https://dana.org/uploadedFiles/Pdfs/brainimagingtechnologies.pdf

[4] Arora, A. and Bhagat, N. (2016) Insight into the Molecular Imaging of Alzheimer's Disease. International Journal of Biomedical Imaging, 2016, 17.

https://doi.org/10.1155/2016/7462014

[5] Zwergel, C., Stazi, G., Valente, S. and Mai, A. (2016) Histone Deacetylase Inhibitors: Updated Studies in Various Epigenetic-Related Diseases. Journal of Clinical Epigenetics, 2,7 .

[6] Marks, P.A. and Dokmanovic, M. (2005) Histone Deacetylase Inhibitors: Discovery and Development as Anticancer Agents. Expert Opinion on Investigational Drugs, 14, 1497-1511. https://doi.org/10.1517/13543784.14.12.1497

[7] Ceacci, E. and Minucci, S. (2016) Inhibition of Histone Deacetylases in Cancertherapy: Lessons from Leukaemia. British Journal of Cancer, 114, 605-611. https://doi.org/10.1038/bjc.2016.36

[8] Kirkpatrick, B. (2015) HDAC Inhibitor Could Sharpen Memory and Treat Cancer. https://www.whatisepigenetics.com/hdac-inhibitor-could-sharpen-memory-and-tre at-cancer/

[9] Weinhold, B. (2006) Epigenetics: The Science of Change. Environmental Health Perspectives, 114, A160-A167. https://doi.org/10.1289/ehp.114-a160

[10] Volmar, C.H. and Wahlestedt, C. (2015) Histone Deacetylases (HDACs) and Brain Function. Neuroepigenetics, 1, 20-27. https://doi.org/10.1016/j.nepig.2014.10.002

[11] Xu, K., Dai, X.L., Huang, H.C. and Jiang, Z.F. (2011) Targeting HDACs: A Promising Therapy for Alzheimer's Disease. Oxidative Medicine and Cellular Longevity, 2011, Article ID: 143269.

[12] Levenson, J.M. and Sweatt, J.D. (2005) Epigenetic Mechanisms in Memory Formation. Nature Reviews Neuroscience, 6, 108-118. https://doi.org/10.1038/nrn1604

[13] Yang, S.S., Zhang, R., Wang, G. and Zhang, Y.F. (2017) The Development Prospection of HDAC Inhibitors as a Potential Therapeutic Direction in Alzheimer's Dis- 
ease. Translational Neurodegeneration, 6, 19.

https://doi.org/10.1186/s40035-017-0089-1

[14] Lu, X., Wang, L., Yu, C., Yu, D. and Yu, G. (2015) Histone Acetylation Modifiers in the Pathogenesis of Alzheimer's Disease. Frontiers in Cellular Neuroscience, 9, 226. https://doi.org/10.3389/fncel.2015.00226

[15] Selenica, M.L., Benner, L., Housley, S.B., Manchec, B., Lee, D.C., Nash, K.R., Kalin, J., Bergman, J.A., Kozikowski, A., Gordon, M.N. and Morgan, D. (2014) Histone Deacetylase 6 Inhibition Improves Memory and Reduces Total Tau Levels in a Mouse Model of Tau Deposition. Alzheimer's Research \& Therapy, 6, 12.

http://alzres.com/content/6/1/12 https://doi.org/10.1186/alzrt241

[16] Couto, P.J. and Millis, R.M. (2015) PET Imaging of Epigenetic Influences on Alzheimer's Disease. International Journal of Alzheimer's Disease, 2015, Article ID: 575078. https://doi.org/10.1155/2015/575078

[17] Yeh, H.H., Tian, M., Hinz, R., Young, D., Shavrin, A., Mukhapadhyay, U., Flores, L.G., Balatoni, J., Soghomonyan, S., Jeong, H.J. and Pal, A. (2013) Imaging Epigenetic Regulation by Histone Deacetylases in the Brain Using PET/MRI with ${ }^{18}$ F-FAHA. Neuroimagine, 64, 630-639. https://doi.org/10.1016/j.neuroimage.2012.09.019

[18] Mottamal, M., Zheng, S., Huang, T.L. and Wang, G. (2015) Histone Deacetylase Inhibitors in Clinical Studies as Templates for New Anticancer Agents. Molecules, 20, 3898-3941. https://doi.org/10.3390/molecules20033898

[19] Li, M.H., Shiue, C.Y., Chang, H.C. and Chu, H.H. (2017) Compounds of Imaging Agent with HDAC Inhibitor for Treatment of Alzheimer Syndrome and Method of Synthesis Thereof. US9, 603, 950B1.

[20] Li, M.H., Shiue, C.Y., Chang, H.C. and Chu, H.H. (2018) Compound and Analogs for Tracing Histone Acetylation Inhibitors PET Imaging for Diagnosis and Treatment of Tumors. Patent has been approved by USA and Taiwan.

[21] Portychova, L. and Schug, K.A. (2017) Instrumentation and Applications of Electrochemistry Coupled to Mass Spectrometry for Studying Xenobiotic Metabolism: A Review. Analytical Chimica Acta, 993, 1-21. https://doi.org/10.1016/j.aca.2017.08.050

[22] Chen, W.H. (2017) Determination of the Metabolites of FEONM, an Alzheimer's Disease Radio-Imaging Diagnosis Agent in Various Biosystems Using LC/Tandem Mass Spectrometry. EC Pharmacology and Toxicology, 4, 40-50.

[23] Mammalian Liver Microsomesguidelines for Use, TF000017 Rev 1.0, Supplied by BD Biosciences.

[24] Cyprotex Co. In Vitro “ADME \& PK-Plasma Stability”. http://www.cyprotex.com/admepk/in-vitro-metabolism/plasma-stability

[25] Seto, E. and Yoshida, M. (2014) Erasers of Histone Acetylation: The Histone Deacetylase Enzymes. Cold Spring Harbor Perspective in Biology, 6, a018713. https://doi.org/10.1101/cshperspect.a018713

[26] Kuevi, U.A., Atohoun, G.S.Y., Kpotin, A.G., Kpota-Hounguè, A.T., Burke, L.A. and Mensah, J.B. (2014) DFT Study of Benzamide Coordination with Zinc (II). International Research Journal of Pure \& Applied Chemistry, 4, 339-351. https://doi.org/10.9734/IRJPAC/2014/7645

[27] Kantharaj, E. and Jayaraman, R. (2011) Histone Deacetylase Inhibitors as Therapeutic Agents for Cancer Therapy: Drug Metabolism and Pharmacokinetic Proper- 
ties, Chapter 5. In: Rundfeldt, C., Ed., Drug Development-A Case Study Based Insight into Modern Strategies, InTech, Rijeka, 101-120.

http://www.meipharma.com/sites/default/files/Drug\%20Development\%20\%282011 \%29.pdf 